\title{
Generating Synchrony from the Asynchronous: Compensation for Cochlear Traveling Wave Delays by the Dendrites of Individual Brainstem Neurons
}

\author{
Matthew J. McGinley, ${ }^{1}$ M. Charles Liberman, ${ }^{2}$ Ramazan Bal, ${ }^{1}$ and Donata Oertel ${ }^{1}$ \\ ${ }^{1}$ Department of Neuroscience, School of Medicine and Public Health, University of Wisconsin, Madison, Madison, Wisconsin 53706, and ${ }^{2}$ Massachusetts \\ Eye and Ear Infirmary, Eaton Peabody Laboratory, Boston, Massachusetts 02114
}

\begin{abstract}
Broadband transient sounds, such as clicks and consonants, activate a traveling wave in the cochlea. This wave evokes firing in auditory nerve fibers that are tuned to high frequencies several milliseconds earlier than in fibers tuned to low frequencies. Despite this substantial traveling wave delay, octopus cells in the brainstem receive broadband input and respond to clicks with submillisecond temporal precision. The dendrites of octopus cells lie perpendicular to the tonotopically organized array of auditory nerve fibers, placing the earliest arriving inputs most distally and the latest arriving closest to the soma. Here, we test the hypothesis that the topographic arrangement of synaptic inputs on dendrites of octopus cells allows octopus cells to compensate the traveling wave delay. We show that in mice the full cochlear traveling wave delay is $1.6 \mathrm{~ms}$. Because the dendrites of each octopus cell spread across approximately one-third of the tonotopic axis, a click evokes a soma-directed sweep of synaptic input lasting $0.5 \mathrm{~ms}$ in individual octopus cells. Morphologically and biophysically realistic, computational models of octopus cells show that soma-directed sweeps with durations matching in vivo measurements result in the largest and sharpest somatic EPSPs. A low input resistance and activation of a low-voltage-activated potassium conductance that are characteristic of octopus cells are important determinants of sweep sensitivity. We conclude that octopus cells have dendritic morphologies and biophysics tailored to accomplish the precise encoding of broadband transient sounds.
\end{abstract}

\section{Introduction}

Broadband transients are prominent in natural sounds, including in human speech. Such sounds activate the cochlea as a traveling wave that starts at its base and ends at the apex (see Fig. $1 A$, inset). In auditory nerve fibers, the latency to firing is shortest for fibers tuned to high frequencies and longer for fibers tuned to lower frequencies. In many mammals, including humans, latency differences in responses to high and low frequencies can reach $8 \mathrm{~ms}$ (Ruggero and Rich, 1987). It is not understood how central auditory neurons and neuronal circuits represent complex sounds in which the encoding of the spectral components is temporally dispersed.

Auditory nerve fibers convey acoustic information to several groups of principal cells in the ventral cochlear nucleus (VCN) (see Fig. 1B). One of these, octopus cells, receives input from large numbers of auditory nerve fibers $(>60$ in mice) that are

Received Jan. 18, 2012; revised April 30, 2012; accepted May 9, 2012.

Author contributions: M.J.M. and D.0. designed research; M.J.M., M.C.L., and R.B. performed research; M.J.M. and M.C.L. analyzed data; M.J.M. and D.0. wrote the paper.

This work was supported by NIH Grant DC00176. We thank Inge Siggelkow for histology, Yaniv Lazimy for Neurolucida reconstructions, Andrew Davison for simulated annealing code, Bill Kath for help learning NEURON, John Rinzel and Michiel Remme for NEURON code for potassium channels, Michael Migliore for NEURON code for HCN channels, and Meyer Jackson, Larry Trussell, Kevin Bender, Megan Hagenauer, and Flavio Frohlich for helpful comments on this manuscript.

Correspondence should be addressed to Matthew J. McGinley, Department of Neurobiology, Yale University, New Haven, CT 06510. E-mail: matthew.mcginley@yale.edu.

DOI:10.1523/JNEUROSCI.0272-12.2012

Copyright $\odot 2012$ the authors $\quad 0270-6474 / 12 / 329301-11 \$ 15.00 / 0$ tuned to a wide range of sound frequencies, yet fire rapidly, reliably, and with extraordinary temporal precision (Oertel et al., 2000; Smith et al., 2005).

Synaptic and intrinsic biophysical properties contribute to the temporal precision in firing of octopus cells. Octopus cells have brief synaptic currents (Gardner et al., 1999; Cao and Oertel, 2010). They also have a large, low-voltage-activated $\mathrm{K}^{+}$conductance $\left(g_{\mathrm{KL}}\right)$ that is balanced by an opposing hyperpolarizationactivated conductance $\left(g_{\mathrm{h}}\right)$, giving them a short membrane time constant $(\sim 0.3 \mathrm{~ms})$ and low input resistance $(\sim 5 \mathrm{M} \Omega)$ (Golding et al., 1995, 1999; Bal and Oertel, 2000, 2001; Cao and Oertel, 2011). If an EPSP does not exceed a threshold rate of depolarization $(>10 \mathrm{mV} / \mathrm{ms})$, the activation of $g_{\mathrm{KL}}$ repolarizes EPSPs and prevents firing (Ferragamo and Oertel, 2002).

Octopus cells were named for having dendrites that emanate unidirectionally from their cell bodies, rostralward in mice, across the tonotopic array of the terminals of auditory nerve fibers (Osen, 1969). They are prominent in all mammals, including humans (Adams, 1986, 1997). In mice, those auditory nerve fibers that encode the lowest frequencies lie most caudally and those that encode the highest frequencies lie most rostrally (see Fig. 1 B) (Wickesberg and Oertel, 1988). The dendrites of octopus cells receive input regarding the highest frequencies, those with the shortest traveling wave delays, at their distal tips and regarding the lowest frequencies, those with the longest traveling wave delays, most proximally (see Fig. 1 B, inset) (Golding et al., 1995). Broadband transient sounds thus activate an octopus cell with a sweep of excitation that spreads from the distal tips of its den- 
drites toward the soma (see Fig. $1 B$, inset, yellow arrow). We test the hypothesis that octopus cells are tuned to respond to these sweeps. Some of these results have previously been presented in preliminary form (McGinley et al., 2005).

\section{Materials and Methods}

In vivo measurement of traveling wave delays. Recordings were made from single auditory nerve fibers from $\mathrm{CBA} / \mathrm{Ca}$ J mice of either sex between 8 and 17 weeks of age. Mice were anesthetized with $5 \mathrm{mg} / \mathrm{kg}$ xylazine and $1.32 \mathrm{mg} / \mathrm{kg}$ urethane and were kept warm with a heating pad and by keeping the experimental chamber warm. The cartilaginous ear canals were removed, the scalp was reflected, the skull was opened, and a semicerebellectomy was performed to expose the cochlear nucleus. Glass microelectrodes filled with $2 \mathrm{M} \mathrm{KCl}$ and $4 \%$ methyl blue were inserted into the cochlear nucleus within $1 \mathrm{~mm}$ of the edge of the temporal bone, angled $24^{\circ}$ in the sagittal plane and laterally at $10^{\circ}$ in the coronal plane. These procedures were approved by the Institutional Animal Care and Use Committee of the Massachusetts Eye and Ear Infirmary.

Dual electrostatic sound sources (TDT; ED-1) and a Knowles electrets microphone were coupled to a probe tube. The sensitivity of the probe-tube microphone was calibrated for frequencies between 1.0 and $73 \mathrm{kHz}$ using a calibrated Bruel \& Kjær 1/4 inch condenser microphone in a coupler. Single units were found with noise bursts. Auditory nerve fibers were distinguished from cochlear nuclear units based on latency, coefficient of variation, interspike intervals, and peristimulus time histograms. Tone bursts of $50 \mathrm{~ms}$ duration with $2.5 \mathrm{~ms}$ rise and fall times were presented at 10/s. Each data point is the mode of the first spike latency in 200 repetitions of responses to tone bursts $30 \mathrm{~dB}$ above threshold at the best frequency.

In vitro recordings of octopus neurons. Coronal slices $(250 \mu \mathrm{m})$ of the posterior VCN were made from 15- to 17-d-old ICR mice of either sex, using procedures approved by the Institutional Animal Care and Use Committee of the University of Wisconsin. Slices were cut, maintained, and recorded at $33^{\circ} \mathrm{C}$ in carbogenated saline composed of the following (in mM): $127 \mathrm{NaCl}, 3 \mathrm{KCl}, 1.2 \mathrm{KH}_{2} \mathrm{PO}_{4}, 2.4 \mathrm{CaCl}_{2}, 1.3 \mathrm{MgSO}_{4}, 3 \mathrm{HEPES}$, $20 \mathrm{NaHCO}_{3}$, and 10 glucose, $\mathrm{pH} 7.4$ (Bal and Oertel, 2000).

Whole-cell recordings in voltage or current clamp were made using a Multiclamp 700B amplifier (100 kHz sample rate; $20-24 \mathrm{kHz}$ low-pass filter cutoff). Borosilicate pipettes ( $4.0-4.8 \mathrm{M} \Omega$ in the bath) were coated with Sylgard along the shaft and close to the tip and then fire polished. Capacitance compensation in the cell-attached configuration had a fast component, $C_{\mathrm{pf}}=6.2 \pm 0.7 \mathrm{pF}, \tau_{\mathrm{pf}}=0.95 \pm 0.05 \mu \mathrm{s}$, and a smaller slow component, $C_{\mathrm{ps}}=1.0 \pm 0.3 \mathrm{pF}, \tau_{\mathrm{ps}}=75 \pm 7 \mu \mathrm{s}(=7)$ that could affect the speed of the clamp. Some of the fast component was present without a pipette attached, $C_{\mathrm{amp}}=2.271 \pm 0.005 \mathrm{pF}, \tau_{\mathrm{amp}}=0.857 \pm 0.007 \mu \mathrm{s}$, and therefore occurred in the amplifier and/or head stage. The pipette solution in all experiments consisted of the following (in $\mathrm{mM}$ ): 110 K-gluconate, $4.5 \mathrm{MgCl}_{2}$, 9 EGTA, 9 HEPES, $4 \mathrm{Na}_{2} \mathrm{ATP}, 0.3 \mathrm{NaGTP}, 14$ phosphocreatine, $\mathrm{pH} 7.4$, and $1 \%$ biocytin. Octopus cells were identified by their anatomical location, low input resistance, and response to depolarizing current steps. Labeling with biocytin confirmed the physiological identification in all cases.

Voltage-clamp recordings were made with or without whole-cell capacitance and series resistance compensation. Current-clamp recordings were performed with and without bridge balance correction, and the pipette capacitance was compensated based on the $C_{\mathrm{pf}}$ measured cellattached. Voltage-clamp data were not used for simulations because the

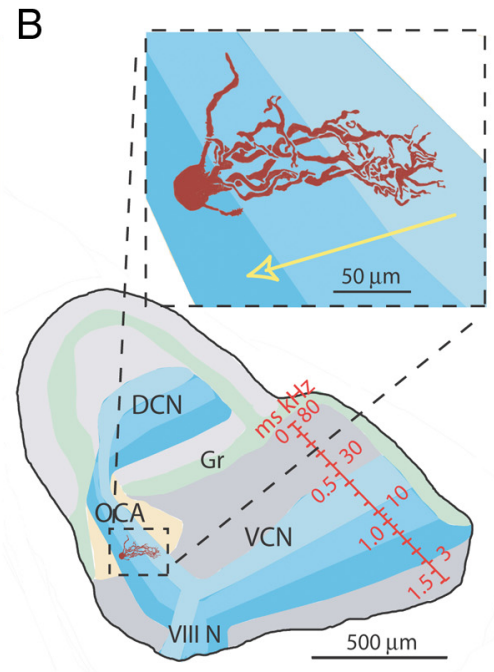

est Frequency $(\mathrm{kHz})$ 
$g_{\mathrm{H}}=G_{\mathrm{H}} *\left(h_{1} *\right.$ frac $+h_{2} *(1-$ frac $\left.)\right)(2.2) ; \frac{d h_{1}}{d t}=\left(h_{\text {inf }}-h_{1}\right) / \tau_{1}(2.3) ;$ $\frac{d h_{2}}{d t}=\left(h_{\text {inf }}-h_{2}\right) / \tau_{2}(2.4) ; \tau_{1}=\beta_{1} /\left(q_{\mathrm{t}} * \mathrm{al}_{\mathrm{h}} *\left(1+\alpha_{1}\right)\right)(2.5) ; \tau_{2}=\beta_{2} /$ $\left(q_{\mathrm{t}} * \mathrm{a} 2_{\mathrm{h}} *\left(1+\alpha_{2}\right)\right)(2.6) ; h_{\mathrm{inf}}=1 /\left(1+e^{\left.\left(v-\mathrm{th}_{\mathrm{inf}}\right) / \mathrm{ginf}\right)}\right.$ (2.7); $\alpha_{1}=e^{\left(v-\eta_{\text {hatit }}\right) * C_{\text {emp }}}(2.8) ; \alpha_{2}=e^{\left(v-\eta_{\text {hafr }}\right) * C_{\text {eemp }}}(2.9) ; \beta_{1}=e^{\left(v-\eta_{\text {laft }}\right) * 0.3 * C_{\text {eemp }}}$ (2.10); $\beta_{2}=e^{\left(v-\eta_{\text {afta }}\right) * 0.6 * C_{\text {temp }}}(2.11) ; C_{\text {temp }}=\frac{0.003 * 9.648 e 4}{8.314 *(273.16+\text { cel })}(2.12)$; $q_{t}=q_{10} *(\mathrm{cel}-33) / 10(2.13)$. The temperature (cel) in active models was $35^{\circ} \mathrm{C}$ and the $q_{10}$ was 4.5 (Cao and Oertel, 2005). The ion channel kinetics might be slightly faster in vivo due to a higher temperature. Input resistances for active models with scaled membrane conductance $(1 / 3 g, 1 / 10 g$, etc.) were calculated using the average small voltage response to a hyperpolarizing or depolarizing current step in each model. Current steps were as follows (in pA): $500 \pm 100$ (1 $g) ; 260 \pm 30(1 / 3 g) ; 120 \pm 10(1 / 10 g) ; 50 \pm 3(1 / 30 g) ; 19 \pm 4(1 / 100 g)$.

For EPSP simulations, synaptic inputs were modeled as a sum of two exponentials, with a rising time constant of $100 \mu \mathrm{s}$, a decay time constant of $300 \mu \mathrm{s}$, and a reversal potential of $0 \mathrm{mV}$ (Gardner et al., 1999). The resting potential in passive models was set to $-63 \mathrm{mV}$. The peak amplitude of the conductance of individual synapses in each model was set so that the composite somatic EPSP was $15 \mathrm{mV}$ for zero sweep duration (see Fig. $5 F$ ). The activation time of each synapse was increased linearly as a function of the distance from the soma, with the most distal synapse having the maximal delay. The sweep duration thus corresponds to this maximum delay. For each synapse, $g_{\text {syn }}(t)=g_{\max } \cdot 1 / \eta \cdot\left(-e^{-t / \tau_{r}}+e^{-/ \tau_{d}}\right)$, where $g_{\text {max }}$, peak synaptic conductance; $\eta$, normalization factor; $\tau_{\mathrm{r}}, \tau$ rise (in milliseconds); and $\tau_{\mathrm{d}}, \tau$ decay (in milliseconds). The normalization factor $\eta=-e^{-t_{p} / \tau_{r}}+e^{-t_{p} / \tau_{d}}$, where $t_{\mathrm{p}}=\log \left(\tau_{\mathrm{d}} / \tau_{\mathrm{r}}\right) \cdot\left(\tau_{\mathrm{d}} \cdot \tau_{\mathrm{r}}\right) /\left(\tau_{\mathrm{d}}-\tau_{\mathrm{r}}\right)$ is the time corresponding to peak synaptic conductance. Rate of depolarization $(d V / d t)$ and integration window (duration) of the somatic EPSP were calculated for $10-90 \%$ of the rising phase. Fast amplitude percentage was calculated as $\left(100^{*} A_{\mathrm{f}} /\left(A_{\mathrm{f}}+A_{\mathrm{s}}\right)\right)$, where $A_{\mathrm{f}}$ and $A_{\mathrm{s}}$ are the amplitude coefficients of the fast and slow exponentials, respectively, of a double-exponential fit.

Morphological reconstructions. Recorded octopus neurons were filled with biocytin through the patch pipette. After the end of the recordings, slices were fixed in $0.4 \%$ paraformaldehyde for $24 \mathrm{~h}$, embedded in a mixture of gelatin and egg albumin, resectioned at $60 \mu \mathrm{m}$, processed to visualize biocytin (Vectastain $\mathrm{ABC}$ kit), and counterstained with cresyl violet. For seven neurons, a digital reconstruction of the morphology of the cell was created using Neurolucida and analyzed with Neuroexplorer (MicroBrightField). Digital morphologies were imported into NEURON (Hines and Carnevale, 1997) using CVAPP (www.compneuro.org). Digital morphologies were not corrected for $z$-axis shrinkage. Image of the mouse cochlea in Figure $1 \mathrm{~A}$, inset, is from a lacZ-NT3 reporter mouse (Sugawara et al., 2007).

Statistics. Data are presented as mean \pm SD, except as noted in Figure 4. Repeated-measures ANOVA (rmANOVA) followed by Tukey's post hoc was used for comparisons between models, and the $F$ statistic and group $p$ value are reported. Within-model comparisons were made using $t$ tests, and reported $p$ values were adjusted for multiple comparisons using a Šidák correction as follows: $\alpha=1-(1-\bar{a})^{n}$, where $\bar{a}$ is the uncorrected $p$ value, and $n$ is the number of models compared. $R^{2}$ values are the Pearson correlation coefficient, and associated $p$ values test the null hypothesis of no correlation. Statistics were performed with OriginPro 8 (OriginLab Corporation).

\section{Results}

\section{The traveling wave delay in mice}

Responses to clicks were used to measure traveling wave delays in auditory nerve fibers in mice. The latency from the presentation of the click to the mode of the earliest spike was plotted as a function of the characteristic frequency of the fiber (Fig. 1A). All fibers are subject to conduction delays of $\sim 1 \mathrm{~ms}$ that include synaptic transmission from hair cells and propagation of action potentials out of the cochlea and into the cochlear nuclei. Traveling wave delays are those delays that vary with characteristic frequency (Fig. $1 A$, solid red line). Traveling wave delays spanned

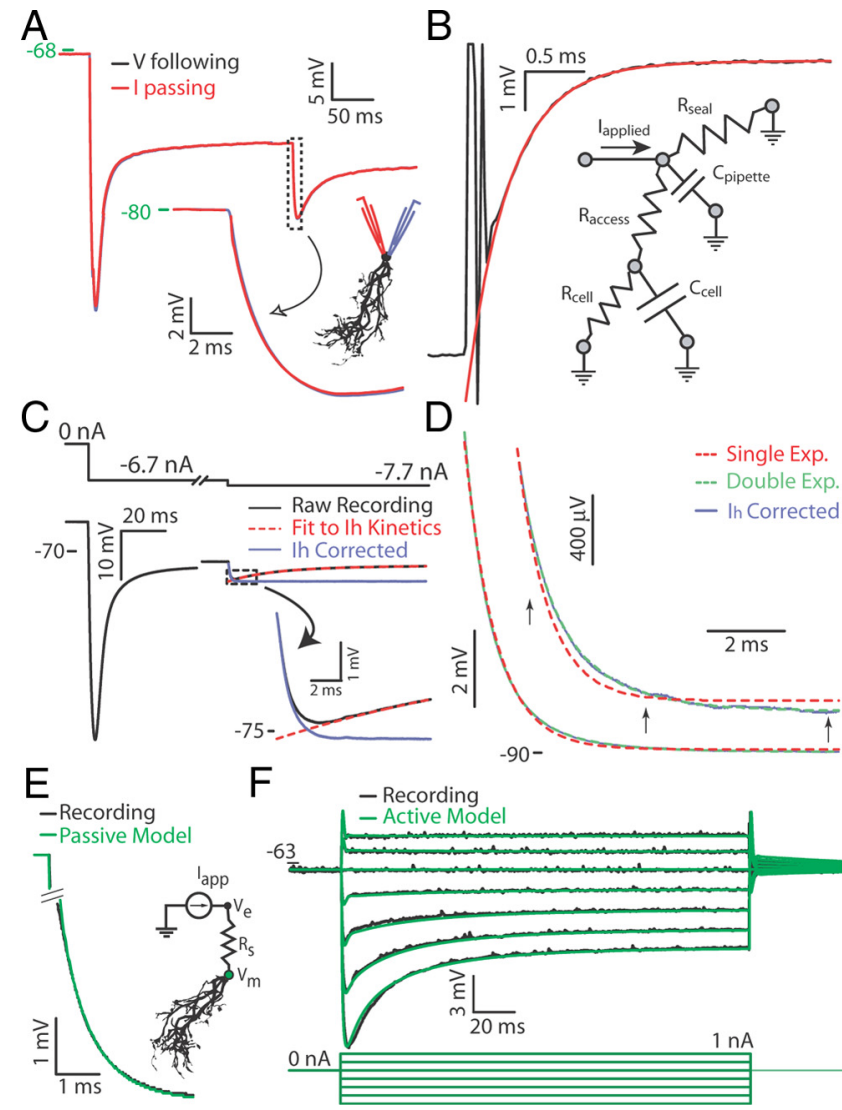

Figure 2. Constructing biophysical models of octopus cells. $A$, Superimposed voltage responses from the current passing (red) and voltage following (black) electrodes from an example dual-patch recording, illustrated at the inset (bottom right). The current was stepped from 0 to $-3 \mathrm{nA}$, and then to $-4 \mathrm{nA}$ while zero current was applied through the voltage monitoring electrode. The response of the current passing electrode was corrected with a small DC offset $(0.6 \mathrm{mV})$ due to zeroing error, and corrected for a series resistance of $8.1 \mathrm{M} \Omega$ so that traces were aligned at the steady state before the second current step. The voltage changes in response to the second step are shown at higher magnification. $\boldsymbol{B}$, Response of the amplifier used in recordings (Multiclamp 700B) when attached to a hardware circuit representing octopus cells, to a current step (black trace) with a superimposed single exponential fit (red trace). A diagram of the hardware circuit is shown, which represents the wholecell configuration of an octopus cell in current clamp. A circuit with only the seal resistance and pipette capacitance was also used, representing the cell-attached configuration. C, The response of an octopus neuron to a hyperpolarizing current step exhibited a deep sag back toward rest (bottom left) resulting from the activation of $I_{h}$. A second hyperpolarizing current step was then applied (middle). The response to the second current step exhibited a passive decay followed by further activation of $l_{h}$. The slow activation of $l_{h}$ was fit with a sum of two exponentials (red dashed curves). Voltage responses were corrected off-line for $8.5 \mathrm{M} \Omega$ series resistance. The break during the first $400 \mathrm{~ms}$ hyperpolarizing step was $340 \mathrm{~ms}$; timescales are the same before and after the break. The inset shows the region to which passive transients were fit (dashed box) at higher magnification. $D$, Example of a typical $/ h^{-}$ corrected passive transient (blue curves) is superimposed on single-exponential (red dashed curves) and a double-exponential fit (green dashed curves). The inset (indicated by arrows) shows the time course of voltage change at a higher voltage resolution on the same timescale. The two exponentials had a fast time constant of $0.61 \mathrm{~ms}$ and a slow time constant of $1.6 \mathrm{~ms}$, and fast percentage of 91 . The single-exponential fit had a time constant of $0.81 \mathrm{~ms}$. E, An example recording, corrected for $I_{\mathrm{h}}$ (black curve), and the superimposed best fit passive simulation (green curve). A section of the trace that contained the series resistance drop, indicated by the break, was removed. The inset shows a diagram of the circuit used for passive simulations: $I_{\text {app }}$ applied current; $V_{\mathrm{e}^{\prime}}$ electrode voltage; $R_{\mathrm{s}^{\prime}}$ series resistance; $V_{m^{\prime}}$ membrane voltage. $\boldsymbol{F}$, Response of an octopus cell (top black traces) to a family of long current steps (bottom traces). The superimposed green traces show the responses to current steps of the same magnitude in an active compartmental model of the same cell.

a range of $1.6 \mathrm{~ms}$ across the hearing range of mice (Fig. $1 \mathrm{~A}$, dashed red lines). These measurements also helped to define the tonotopic map in mice. The range of characteristic frequencies has been measured in mice (Taberner and Liberman, 2005). 
Table 1. Optimized passive model parameters and associated errors in the model

\begin{tabular}{|c|c|c|c|c|c|c|c|c|c|c|}
\hline & & & & & & & & & & Best fit \\
\hline$R_{\mathrm{i}}\left(\Omega^{*} \mathrm{~cm}\right)$ & 120 & 120 & 120 & 200 & 200 & 200 & 250 & 250 & 250 & $195 \pm 88$ \\
\hline$C_{m}\left(\mu \mathrm{F} / \mathrm{cm}^{2}\right)$ & 1.2 & 2 & 2.8 & 1.2 & 2 & 2.8 & 1.2 & 2 & 2.8 & $2.1 \pm 0.9$ \\
\hline$G_{\mathrm{ms}}\left(S / \mathrm{cm}^{2}\right)$ & $6.9 \pm 4.2$ & $3.1 \pm 4.1$ & $1.5 \pm 2.1$ & $7.7 \pm 2.7$ & $4.2 \pm 4.1$ & $2.8 \pm 3.3$ & $7.7 \pm 2.4$ & $4.9 \pm 3.9$ & $3.3 \pm 3.8$ & $7.2 \pm 6.8$ \\
\hline$G_{m d}\left(S / \mathrm{cm}^{2}\right)$ & $0.0010 \pm 0.0003$ & $0.0021 \pm 0.0004$ & $0.0028 \pm 0.0003$ & $0.00096 \pm 0.0003$ & $0.0019 \pm 0.0004$ & $0.0027 \pm 0.0004$ & $0.0009 \pm 0.0003$ & $0.0018 \pm 0.0004$ & $0.0026 \pm 0.0005$ & $0.0019 \pm 0.0010$ \\
\hline$G_{\text {ratio }}$ & $8800 \pm 9100$ & $1800 \pm 2400$ & $505 \pm 705$ & $9300 \pm 6000$ & $2600 \pm 2800$ & $1100 \pm 1300$ & $9600 \pm 5700$ & $3200 \pm 2900$ & $1510 \pm 1700$ & $10,200 \pm 1700$ \\
\hline Error $\left(R^{2}\right)$ & $0.00062 \pm 0.00064$ & $0.0033 \pm 0.0059$ & $0.011 \pm 0.017$ & $0.00054 \pm 0.00039$ & $0.00087 \pm 0.00115$ & $0.0035 \pm 0.0062$ & $0.00060 \pm 0.00029$ & $0.00052 \pm 0.00029$ & $0.0019 \pm 0.0023$ & $0.00037 \pm 0.00030$ \\
\hline $\operatorname{Rs}(M \Omega)$ & $6.1 \pm 0.6$ & $6.8 \pm 0.7$ & $7.4 \pm 0.8$ & $5.1 \pm 0.5$ & $5.8 \pm 0.6$ & $6.4 \pm 0.6$ & $4.6 \pm 0.4$ & $5.4 \pm 0.6$ & $6.0 \pm 0.7$ & $6.0 \pm 0.8$ \\
\hline $\operatorname{Ri}(M \Omega)$ & $4.8 \pm 0.8$ & $4.1 \pm 1.0$ & $3.5 \pm 1.1$ & $5.8 \pm 0.7$ & $5.1 \pm 0.8$ & $4.5 \pm 0.8$ & $6.3 \pm 0.6$ & $5.5 \pm 0.8$ & $4.9 \pm 1.0$ & $4.9 \pm 1.1$ \\
\hline
\end{tabular}

Cytoplasmic resistivity $\left(R_{\mathrm{i}}\right)$ and specific membrane capacitance $\left(C_{\mathrm{m}}\right)$ were constrained to all combinations of a high, middle, and low value (left columns) or the best fit set of parameters (right-most column). Low errors are found throughout this relatively large region of parameter space surrounding the best fit parameter set. Constraining the cytoplasmic resistivity to 90 or $300 \Omega^{*} \mathrm{~cm}$ resulted in worse fits (data not shown).

Table 2. Parameters in the active model

\begin{tabular}{|c|c|c|c|c|c|c|c|c|c|}
\hline$\overline{i_{\mathrm{KL}}}$ & $\begin{array}{c}G_{\mathrm{KL}}\left(\mathrm{kS} / \mathrm{cm}^{2}\right) \\
45 \pm 7\end{array}$ & $\begin{array}{l}w_{\text {half }}(\mathrm{mV}) \\
62.0 \pm 1.2\end{array}$ & $\begin{array}{l}w_{q}(\mathrm{mV}) \\
6.8 \pm 1.5\end{array}$ & $\begin{array}{l}z_{\text {half }}(\mathrm{mV}) \\
64.0 \pm 2.7\end{array}$ & $\begin{array}{l}z_{q}(\mathrm{mV}) \\
5.0 \pm 1.2\end{array}$ & $\begin{array}{l}w_{\tau, \text { inf }}(\mathrm{ms}) \\
0.48\end{array}$ & $\begin{array}{l}e_{\mathrm{k}}(\mathrm{mV}) \\
-77\end{array}$ & & \\
\hline$i_{\mathrm{H}}$ & $G_{H}\left(\mathrm{kS} / \mathrm{cm}^{2}\right)$ & $\mathrm{a} 1_{\mathrm{h}}\left(\mathrm{ms}^{-1}\right)$ & $\mathrm{a} 2_{\mathrm{h}}\left(\mathrm{ms}^{-1}\right)$ & frac & th $_{\text {inf }}(\mathrm{mV})$ & $q_{\text {inf }}(\mathrm{mV})$ & $e_{\mathrm{H}}(\mathrm{mV})$ & $v_{\text {half1 }}(m V)$ & $v_{\text {half2 }}(\mathrm{mV})$ \\
\hline Leak & $\begin{array}{c}5.3 \pm 2.2 \\
G_{\text {pas }}\left(\mathrm{kS} / \mathrm{cm}^{2}\right) \\
0.53 \pm 0.22\end{array}$ & $\begin{array}{l}4.8 \pm 0.5 \mathrm{e}-3 \\
C_{\mathrm{m}}\left(\mu \mathrm{F} / \mathrm{cm}^{2}\right) \\
1.2\end{array}$ & $\begin{array}{l}2.9 \mathrm{e}-3 \\
e_{\text {pas }}(\mathrm{mV}) \\
-73\end{array}$ & $\begin{array}{l}\quad 0.8 \\
R_{\mathrm{i}}\left(\Omega^{*} \mathrm{~cm}\right) \\
150\end{array}$ & $-72.4 \pm 2.1$ & $5.3 \pm 0.5$ & -38 & & -84 \\
\hline
\end{tabular}

Parameters shown with $\pm S D$ differed between models, whereas those without an SD were the same in all models. Parameters for the low-voltage-activated conductance were as follows: $G_{\mathrm{KL}}$, peak conductance; $w_{\text {half }}$, voltage of half-maximal activation; $w_{\mathrm{q}}$, activation slope factor; $z_{\mathrm{hal}}$, voltage of half-maximal inactivation; $z_{\mathrm{q}}$, inactivation slope factor; $w_{\tau \text { - inf }}$, rate constant; $e_{\mathrm{k}}$, reversal potential. Parameters for the hyperpolarization-activated mixed-cation conductance were as follows: $G_{H}$, peak conductance; $1_{h}$, rate constant for gate $1 ;$ a $2_{h}$, rate constant for gate 2 ; frac, fractional contribution of gate 1 ; th ${ }_{\text {inf }}$, voltage of half-maximal activation; $q_{\text {inf }}$, activation slope factor; $e_{H}$, reversal potential; $v_{\text {half }}$, voltage of half-activation for the rate constant for gate $1 ; v_{\text {half }}$, voltage of half-activation for the rate constant for gate 2 . Leak parameters have the same definition as in passive models. The resting potential was $-63.4 \pm$ $1.0 \mathrm{mV}$, which matched recorded values $(-63.2 \pm 0.7 \mathrm{mV} ; n=4)$.

Characteristic frequencies are arranged along the cochlea as a function of $\log$ frequency; extrapolation to the ends of the cochlea led to estimates of the range of best frequencies from 4.8 to 79.1 $\mathrm{kHz}$ (Müller et al., 2005). Our measurements show fibers with characteristic frequencies as low as $3 \mathrm{kHz}$ (Fig. $1 A$ ). Each octopus neuron spans approximately one-third of the tonotopic array in the cochlear nucleus (Fig. $1 B$, left and inset). Mapping the range of characteristic frequencies and traveling wave delays onto the cochlear nucleus of mice (Fig. $1 B$, red lines) predicted that each octopus neuron will experience $\sim 0.5 \mathrm{~ms}$ of traveling wave delay in its dendrites.

\section{Active and passive models of octopus cells}

To determine how synaptic integration is shaped by the cochlear traveling wave delay, we constructed morphologically and biophysically accurate models of the subthreshold regime of octopus cells. Dual-electrode whole-cell recordings from individual octopus cells indicated that membrane responses can be measured accurately in current clamp despite the very low input resistance of octopus neurons. The current passing electrode very closely follows the monitoring electrode, indicating that distortions resulting from passing current were minimal (Fig. 2A).

Recordings from hardware circuits indicated that the current clamp was able to resolve the extremely rapid membrane responses of octopus cells (Fig. $2 \mathrm{~B}$ ). In the hardware model, circuit element values were as follows: $R_{\text {seal }}, 3 \mathrm{G} \Omega$; $C_{\text {pipette }}, 1.5 \mathrm{pF} ; R_{\text {access }}$, $10 \mathrm{M} \Omega ; R_{\text {cell }}, 4 \mathrm{M} \Omega ; C_{\text {cell }}, 82 \mathrm{pF}$. In voltage clamp, the pipette compensation values obtained from the cell-attached circuit were $4.8 \mathrm{pF}(\tau=0.74 \mu \mathrm{s})$ and $0.38 \mathrm{pF}(\tau=152 \mu \mathrm{s})$. Estimates of the whole-cell parameters with the correction circuitry were as follows: $R_{\text {access }}, 9.5 \mathrm{M} \Omega ; R_{\text {cell }}, 5.9 \mathrm{M} \Omega ; C_{\text {cell }}, 71.8 \mathrm{pF}$, with a bandwidth of $16.2 \mathrm{kHz}$, and $85 \%$ compensation, corresponding to the actual values with modest error. A fit to the current response to a voltage step had a time constant of $261 \mu \mathrm{s}$, in reasonable agreement with the theoretical value of $234 \mu$ s, which was obtained by solving Kirchoff's laws for the circuit (data not shown). This theoretical value is approximately equal to $C_{\text {cell }} *\left[1 / R_{\text {access }}+\right.$ $\left.1 / R_{\text {cell }}\right]=234.3 \mu \mathrm{s}$. The time constant of the exponential fit (red trace) to the voltage response (black trace) for a current step (Fig.
2B) had a time constant of $365 \mu \mathrm{s}$, after a correction for the pipette and amplifier capacitance of $4.8 \mathrm{pF}$. The solution of Kirchoff's laws (data not shown) predicted two time constants equaling 14.7 and $334 \mu \mathrm{s}$, which are approximately equal to $C_{\text {pipette }}{ }^{\star} R_{\text {access }}(15 \mu \mathrm{s})$ and $C_{\text {cell }}{ }^{\star} R_{\text {cell }}(328 \mu \mathrm{s})$, respectively. Therefore, the measured time constant $(365 \mu \mathrm{s})$ was in reasonable agreement with the predicted slow (membrane) time constant $(334 \mu \mathrm{s})$. The fast time constant of the pipette $(14.7 \mu \mathrm{s})$ could not be resolved because it was faster than, and therefore convolved with, the speed of the clamp. Therefore, even though the membrane resistance of octopus neurons $(\sim 4 \mathrm{M} \Omega)$ is $\sim 2$-fold lower than access resistances obtained $(\sim 9 \mathrm{M} \Omega)$, these resistances are easily distinguished by the amplifier due to the $\sim 20$-fold different capacitive loads on the resistors, albeit with a $\sim 10 \%$ error due to amplifier distortions. The dendritic charging components are easier to resolve because they are slower.

Whole-cell recordings were made from 35 anatomically labeled octopus cells in slices from the VCN of mice (Fig. $1 B$ ). Four octopus cells with the best morphologies and recordings were reconstructed digitally and used in simulations. Passive biophysical models were constructed by measuring charging transients in response to long current steps (Fig. 2C) from a holding potential of $-75.4 \pm 4.1 \mathrm{mV}(n=10 ;-6.5 \pm 0.6 \mathrm{nA}$ holding current), which is outside the activation range of $g_{\mathrm{KL}}$, but within the activation range of $g_{\mathrm{h}}$ (Bal and Oertel, 2000, 2001). The activation of $I_{\mathrm{h}}$ was slow, and in the opposite direction of the passive components so the $I_{\mathrm{h}}$ sag could be fit with a sum of two exponentials and subtracted (Fig. 2C). Therefore, traces corrected for $I_{\mathrm{h}}$ contained the passive charging components (Fig. 2D). Passive transients in responses to hyperpolarizing current steps $(1.8 \pm 0.5 \mathrm{nA})$ resulted in a drop of $9.3 \pm 1.0 \mathrm{mV}$ that was well fit by a sum of 2 , but not 1 , exponential functions (Fig. $2 D ; n=4$ ); the slow component likely reflected dendritic charging (Rall et al., 1992). Smaller hyperpolarizing $(1.2 \pm 0.5 \mathrm{nA})$ or depolarizing $(1.0 \pm 0.1 \mathrm{nA})$ steps gave similar results (data not shown) $(n=6)$, confirming that the passive regime was targeted effectively, and subsequent results were pooled. For hyperpolarizing current steps starting from $-75.4 \pm 4.1$, the $I_{\mathrm{h}}$ sag had an amplitude of $3.8 \pm 4.6 \mathrm{mV}$ with a fast time constant of $20.3 \pm 3.1 \mathrm{~ms}$ and a slow time con- 
A
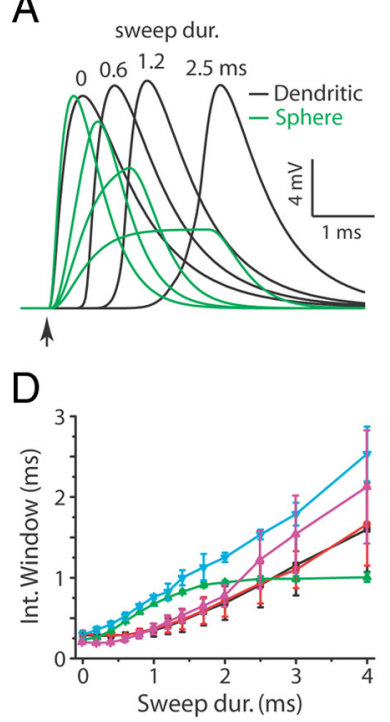

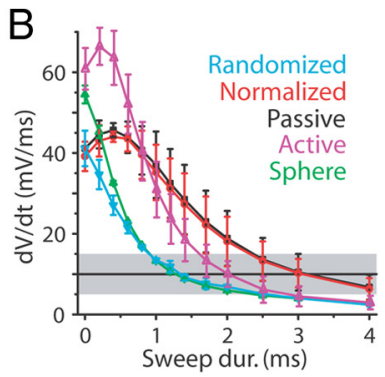

$\mathrm{E}$

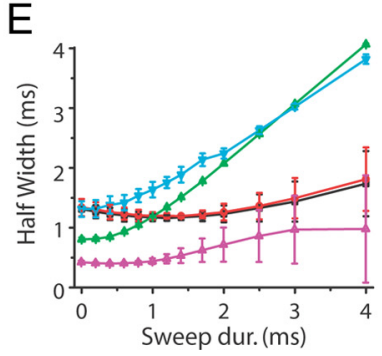

C

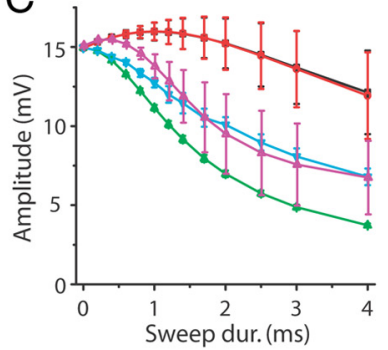

$\mathrm{F}$

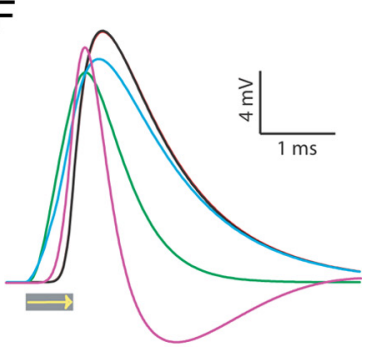

Figure 3. Soma-directed sweeps of excitation produce fast and large EPSPs in octopus cells. $\boldsymbol{A}$, Simulated EPSPs from a passive model octopus cell (black traces) and an isopotential spherical cell model with the same input resistance and membrane time constant (green traces) for four sweep durations (indicated above the EPSPs). The arrowhead indicates the start time of all sweeps. $\boldsymbol{B}$, The rate of depolarization $(d V / d t)$ of simulated somatic EPSPs for active octopus cell models with various sweep durations (magenta triangles), passive octopus models with inputs of uniform amplitudes (black squares), passive models with normalized input amplitudes (red circles), passive models with inputs activated at random times (blue triangles), and passive isopotential sphere models (green triangles). $\boldsymbol{C}-\boldsymbol{E}$, The amplitude, integration window, and half-width of somatic EPSPs, respectively, as a function of sweep duration. The color code is the same as in $\boldsymbol{B}$. $\boldsymbol{F}$, Somatic EPSP in response to a sweep of $0.6 \mathrm{~ms}$ duration, for each of the model types in $\boldsymbol{B}-\boldsymbol{E}$.

A
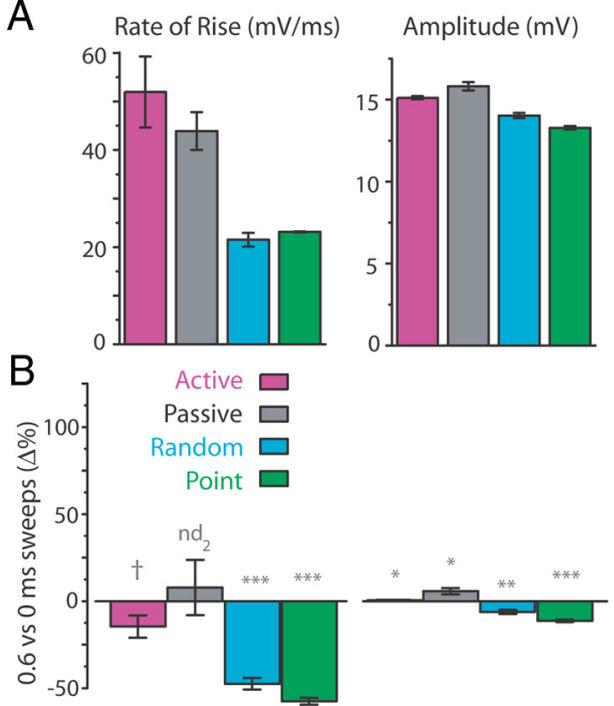
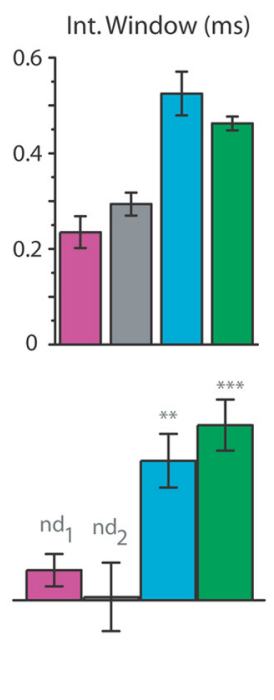

Figure 4. Octopus cell models with dendrites and active channels best compensate traveling wave delays. $A$, A comparison of the shape parameters between models for a sweep duration of $0.6 \mathrm{~ms}$. Statistical comparisons between models are shown in Table 3. $\boldsymbol{B}$, The percentage difference in each shape parameter between EPSPs for 0.6 or $0.0 \mathrm{~ms}$ sweeps. For each model, the percentage difference is compared with the null hypothesis of a zero percentage change, with $p$ values indicated above: ${ }^{*} p<0.05 ;{ }^{* *} p<$ $0.01 ;{ }^{* * *} p<0.005 ; \mathrm{nd}_{1}, p>0.12 ; \mathrm{nd}_{2}, p>0.87 ;{ }^{\dagger} p=0.08$. Statistical comparisons between models are shown in Table 3 . Error bars indicate SEM.

stant of $82 \pm 24 \mathrm{~ms}$. The fast time constant amplitude percentage was $60 \pm 8 \%$. For passive transients from $I_{\mathrm{h}}$-corrected traces, the amplitude was $3.8 \pm 4.6 \mathrm{mV}$ with a fast time constant of $550 \pm 9$ $\mu \mathrm{s}$ and a slow time constant of $1.5 \pm 0.4 \mathrm{~ms}$. The fast time constant amplitude percentage was $91 \pm 5 \%$. The resistance apparent in passive transients in the hyperpolarized range $(4.5 \pm 0.95$ $\mathrm{M} \Omega$ ) was similar to the input resistance measured from the peak
Half Width (ms)

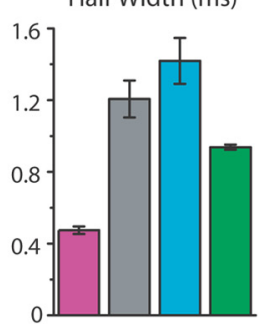

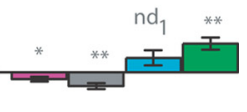

voltages in small current steps from the resting potential $(4.4 \pm 0.7 \mathrm{M} \Omega ; n=8)$. Thus, under these conditions, the overall input conductance resembled that at rest and voltage-dependent changes in input resistance were minimal.

Our morphologically realistic passive model was able to accurately fit the data (Fig. $2 E$ ). The best fit parameters in passive models were as follows $(n=4)$ : membrane conductivity of the soma $\left(G_{\mathrm{ms}}\right)(7.2 \pm 6.8$ $\left.\mathrm{S} / \mathrm{cm}^{2}\right)$, membrane conductivity of the dendrites $\left(G_{\mathrm{md}}\right)\left(1.9 \pm 1.0 \mathrm{mS} / \mathrm{cm}^{2}\right)$, and the resistivity of the intracellular medium $\left(R_{\mathrm{i}}\right)$ $\left(195 \pm 90 \Omega^{*} \mathrm{~cm}\right)$, specific membrane capacitance $\left(C_{\mathrm{m}}\right)\left(2.1 \pm 0.9 \mu \mathrm{F} / \mathrm{cm}^{2}\right)$, and series resistance $\left(R_{\mathrm{s}}\right)(6.0 \pm 0.8 \mathrm{M} \Omega)$. These values for intracellular resistivity correspond well to those from other passive models (Rall et al., 1992; Spruston and Johnston, 1992; Rapp et al., 1994; Mainen et al., 1996; Stuart and Spruston, 1998; Schmidt-Hieber et al., 2007). Furthermore, our estimates of specific membrane capacitance resembled other models (Rapp et al., 1994; Stuart and Spruston, 1998; Thurbon et al., 1998), which are larger than measured values (Gentet et al., 2000) partly due to tissue shrinkage. Forcing the specific membrane capacitance to a value of $1.2 \mu \mathrm{F} / \mathrm{cm}^{2}$ resulted in only small changes in the fits and in the estimates of EPSP shape (see Fig. 6, Table 1).

We constructed active models of octopus cells incorporating $g_{\mathrm{KL}}$ (see Materials and Methods) (Khurana et al., 2011), $g_{\mathrm{H}}$ (see Materials and Methods; http:// senselab.med.yale.edu/modeldb) (Bal and Oertel, 2000), and passive leak ion channels. The parameters of $g_{\mathrm{KL}}$ and $g_{\mathrm{H}}$ were first adjusted to match the average kinetic data from octopus cells (Bal and Oertel, 2000, 2001), and then select parameters were further adjusted so that each compartmental model matched the recordings for voltage responses to a family of long current steps in the neuron with the corresponding morphology (Fig. 2F, Table 2).

\section{Soma-directed sweeps of excitation sharpen the rise of EPSPs}

To determine how traveling wave delays in auditory nerve fiber firing shape the somatic EPSP in octopus cells, we distributed 100 simulated excitatory synaptic currents with equal conductance and realistic kinetics uniformly over the surface area of each simulated octopus cell (see Materials and Methods). We then activated the synapses at times that were a linearly varying function of distance from the soma to produce soma-directed sweeps of excitation of varying duration. In a passive octopus cell model, the somatic EPSP was tall and fast-rising over a wide range of sweep durations (Fig. 3A, black curves). For 
Table 3. Statistical differences between models of EPSP shape parameters

\begin{tabular}{|c|c|c|c|c|c|c|c|c|c|}
\hline \multicolumn{10}{|c|}{ Comparison of the shape parameters for $0.6 \mathrm{~ms}$ sweeps } \\
\hline & Active & Passive & Random & Point & & Active & Passive & Random & Point \\
\hline Active & $\mathrm{mV} / \mathrm{ms} \rightarrow$ & $*$ & $* * *$ & $* * *$ & Active & Int Win $\rightarrow$ & + & $* * *$ & $* * *$ \\
\hline Passive & $* * *$ & & $* * *$ & $* * *$ & Passive & $* * *$ & & $* *$ & $* *$ \\
\hline Random & $* * *$ & $* * *$ & & n.d. & Random & $* * *$ & $* *$ & & $* * *$ \\
\hline Point & $* * *$ & $* * *$ & $* * *$ & $\leftarrow$ Amp & Point & $* * *$ & $* *$ & $* * *$ & $\leftarrow$ Width \\
\hline \multicolumn{10}{|c|}{ Comparison of the percentage change in shape parameters between 0.6 and 0.0 ms sweeps } \\
\hline & Active & Passive & Random & Point & & Active & Passive & Random & Point \\
\hline Active & $\mathrm{mV} / \mathrm{ms} \rightarrow$ & $*$ & $* *$ & $* *$ & Active & Int Win $\rightarrow$ & n.d.2 & $* *$ & $* *$ \\
\hline Passive & $* *$ & & $* *$ & $* *$ & Passive & n.d. ${ }_{1}$ & & $* *$ & $* *$ \\
\hline Random & $* *$ & $* *$ & & n.d. ${ }_{2}$ & Random & $* *$ & $* *$ & & n.d.2 \\
\hline Point & $* *$ & $* *$ & $* *$ & $\leftarrow A m p$ & Point & $* *$ & $* *$ & $* *$ & $\leftarrow$ Width \\
\hline
\end{tabular}

Top, Pairwise comparisons of shape parameters of somatic EPSPs between models for a $0.6 \mathrm{~ms}$ soma-directed sweep of excitation. In each quadrant, two parameters are compared as indicated with the different colors. The $F$ statistic and group $p$ value for rmANOVA were as follows: rate of rise $\left(F_{(1,3)}=656 ; p<1 \mathrm{e}-4\right)$; amplitude $\left(F_{(1,3)}=131,076 ; p<1 \mathrm{e}-8\right)$; integration window $\left(F_{(1,3)}=1396 ; p<5 \mathrm{e}-5\right)$; half-width $\left(F_{(1,3)}=1265 ; p<5 \mathrm{e}-5\right)$. For the pairwise post hoc comparisons: ${ }^{*} p<0.05 ;{ }^{* *} p<0.01 ; * * p<0.001 ;{ }^{\dagger} p=0.06 ; \mathrm{nd}, p=0.88$. Bottom, Pairwise comparisons of the percentage change in shape parameters of somatic EPSPs between a $0.6 \mathrm{~ms}$ soma-directed sweep of excitation with respect to simultaneous excitation. In each quadrant, two parameters are compared, separated by color. For comparisons between models of the percentage change in shape parameters between 0.6 and $0.0 \mathrm{~ms}$ sweeps (bottom entries), the $F$ statistic and group $p$ value for rmANOVA were as follows: rate of rise $\left(F_{(1,3)}=120.5 ; p<0.002\right)$; amplitude $\left(F_{(1,3)}=125.76 ; p<0.002\right)$; integration window $\left(F_{(1,3)}=79.07 ; p<0.003\right)$; half-width $\left(F_{(1,3)}=1265 ; p<5 \mathrm{e}-5\right)$. For the pairwise post hoc comparisons: ${ }^{*} p<0.02 ;{ }^{* *} p<0.002 ; \mathrm{nd}_{1}, p=0.1 ; \mathrm{nd}_{2}, p>0.32$.

comparison, we constructed an isopotential sphere model with the same input resistance and time constant as each octopus cell morphologicaly realistic model. Sphere models also had 100 synapses with the same kinetics and a range of activation times that matched the sweep duration in the corresponding dendritecontaining model. In the sphere model, the EPSP shrank and broadened as the range of activation times was increased (Fig. $3 A$, green curves).

To explore the shaping of EPSPs in more detail, four parameters were calculated for somatic EPSPs in active dendritic, passive dendritic, and passive sphere models: rate of rise (in millivolts/millisecond), amplitude (in millivolts), integration window (in milliseconds), and half-width (in milliseconds). Since octopus cells require fast depolarization to fire action potentials, and thus have narrow integration windows (Ferragamo and Oertel, 2002; Maison et al., 2003; McGinley and Oertel, 2006), we first examined the rate of rise of EPSPs in our models. Active dendritic models achieved the fastest rates of rise for sweep durations $<1$ ms (Fig. 3B, magenta). Passive sphere models had slower rates of rise, which dropped as the sweep duration increased (Fig. $3 B$, green). Passive dendritic models were intermediate (Fig. $3 B$, black). To determine whether the distribution of synapses on dendrites or their somatopetal activation sharpens EPSPs, we created passive dendritic models whose synapses were activated at random times between zero and each corresponding sweep duration. This randomized activation of synapses produced the slowest rates of rise of all the models for all sweep durations (Fig. $3 B$, blue). Therefore, the distal-to-proximal sweep of excitation, not simply the sink effect of the dendrites, is essential for producing rapidly rising EPSPs in octopus cells (Fig. 4A, B, left; Table 3). Rates of rise remained above $d V / d t$ threshold for sweep durations as long as $1.4 \mathrm{~ms}$ in active and $2 \mathrm{~ms}$ in passive models. However, variability in the activation of auditory nerve fibers, in latency for a particular best frequency (Fig. $1 A$ ), and irregularities in morphologies that smear EPSPs, will pull them closer to the $d V / d t$ threshold for a given sweep duration.

Input normalization does not affect sweep sensitivity

In some cortical neurons, an increase in the synaptic conductance with distance from the soma produces somatic miniature EPSPs whose size is independent of the dendritic location of the activated synapse (Magee and Cook, 2000). To evaluate whether such "input normalization" affects the sensitivity to soma-directed sweeps, we performed simulations in which the synaptic conductance increased linearly as a function of distance from the soma. Input normalization was achieved in passive models when the most distal synaptic conductance was $1.8 \pm 0.6$ times the amplitude of proximal synapses. Surprisingly, input normalization had no effect on the dependence of the rate of rise of somatic EPSPs on sweep duration (Fig. 3B, red superimposed on black). Therefore, local depolarization and current spread, not the size of unitary responses at the soma, determine the somatic impact of a synapse during realistic sweeps of synaptic excitation in octopus cells.

\section{EPSPs are sharpest for sweeps matching measured traveling wave delays}

To evoke action potentials, EPSPs in octopus cells must not only have a rapid rise but must also be sufficiently large to reach the voltage activation range for sodium channels. In passive sphere models or passive models with dendrites with randomized activation times, EPSP amplitudes decreased monotonically with sweep duration (Fig. 3C, blue and green). In active models, however, the amplitude of EPSPs had a peak associated with an optimal sweep duration of $0.4 \pm 0.0 \mathrm{~ms}(1.2 \pm 0.4 \mathrm{~ms}$ in passive models) (Figs. $3 C$, black and magenta, $4 A, B$; Table 3 ). Like rates of rise, amplitudes of somatic EPSPs were not affected by input normalization (Fig. 3C, red superimposed on black). The combined effect of sweep duration on the rate of rise and amplitude of EPSPs is captured in the integration window. Integration windows were short in active and passive dendritic models compared with passive sphere or randomized models for sweep durations less than $\sim 2 \mathrm{~ms}$, and shortest for active octopus cell models with sweep durations of $\sim 0.5 \mathrm{~ms}$ (Figs. $3 D, 4 A, B$; Table 3$)$.

Width at half-height (half-width) is a conventional measure of the duration of a waveform that encompasses the rising and falling phases. Half-widths were substantially shorter in active dendritic models than in all other models over the full range of sweep durations explored (Figs. 3E; $4 A, B$, right; Table 3 ). The relationship between integration windows (Fig. $3 D$ ) and halfwidths (Fig. $3 E$ ) indicates that the short half-widths in active octopus cells resulted predominantly from a shorter decay rather than rising phase. Activation of $g_{\mathrm{KL}}$ accelerates the decay phase of 
the EPSP, relieving the slowing effect of dendritic filtering on half-width (Golding et al., 1995, 1999; Oertel et al., 2008; Mathews et al., 2010).

A comparison of EPSP waveforms across models for a sweep duration of $0.6 \mathrm{~ms}$ - approximately the duration of the traveling wave delay-is illustrated in Figure $3 \mathrm{~F}$ and summarized in Figure 4 and Table 3. For this sweep duration, EPSPs in active and passive dendritic models have larger amplitude and faster rises than those in spherical or randomized models. The main differences between passive and active models are a dramatic shortening of the falling phase and a small decrease in integration window and amplitude in the active models, resulting from activation of $g_{\mathrm{KL}}$ during depolarization.

\section{Input resistance controls the optimal sweep duration}

To understand the role of the low input resistance of octopus cells in compensating for traveling wave delays, we created active octopus cell models with the leak conductance, $g_{\mathrm{KL}}$ and $g_{\mathrm{H}}$ values decreased to $1 / 3,1 / 10,1 / 30$, or $1 / 100$ of their best fit values, while all other parameters remained equal. The fastest rate of rise, largest amplitude, shortest integration window, and shortest halfwidth all shifted to longer sweep durations as the input resistance increased (Fig. 5A-D). The rate of rise remained above $d V / d t$ threshold in all models for sweep durations up to 1.7-2 ms (Fig. $5 A$, gray band). In these models, the input resistance increased from $5 \mathrm{M} \Omega$ in best fit models to $110 \mathrm{M} \Omega$ in models for a 100 -fold decrease in channel density. The $d V / d t$ threshold would be expected to decrease as the input resistance increases (McGinley and Oertel, 2006), thus accommodating longer sweep durations in higher input resistance neurons. The integration window for longer sweep durations $(>1 \mathrm{~ms})$ was relatively insensitive to the input resistance (Fig. 5C). Therefore, the amplitude and halfwidth appear to be most important for longer sweeps and higher input resistances.

In active models, the sweep durations that produced the largest, fastest rising EPSPs - the optimal sweep durationswere strongly dependent on the input resistance, increasing from $\sim 0.5$ to $2.0 \mathrm{~ms}$ as the input resistance increased from 5 to $110 \mathrm{M} \Omega$ (Fig. 5E). Passive models, however, were relatively broadly tuned for sweep duration as indicated by their broad error bars (Fig. $5 E$, gray symbols). With a 100 -fold change in membrane conductance, the synaptic conductance necessary to achieve comparable somatic EPSP amplitudes only changed $\sim 3$-fold (Fig. 5F). Thus, the extremely low input resistance of octopus cells can be accommodated with comparatively modest additional synaptic resources.

\section{Ion channel distribution plays a minimal role}

In the best fit passive models, the membrane conductivity was lower in the dendrites than the soma. This is consistent with observations in other passive models (Clements and Redman, 1989; Rapp et al., 1994) and with the measured distribution of $g_{\mathrm{KL}}$ in MSO neurons (Mathews et al., 2010). However, we wondered whether this channel distribution was important for the sensitivity to soma-directed sweeps. Fitting of passive models to voltage traces is not fully constrained, resulting in uncertainty in the estimation of the parameters (Rall et al., 1992). Therefore, we explored the full parameter space that gave reasonable fits, which exhibited a wide range of soma-to-dendrite conductance ratios. Simulations were performed for each octopus cell incorporating all combinations of a high, middle, and low value for $R_{\mathrm{i}}$ and $C_{\mathrm{m}}$ that spanned the range of values that resulted in good fits, which also spanned the range of physiologically reasonable values. For
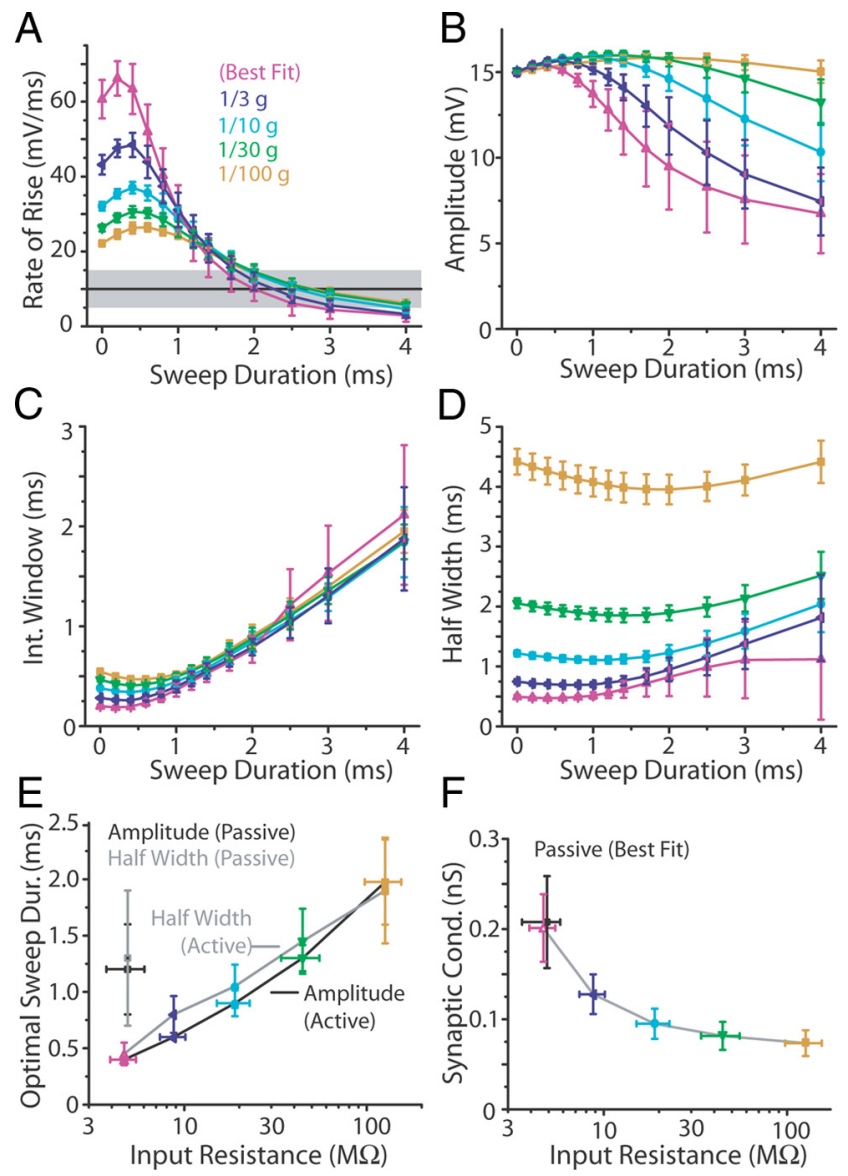

Figure 5. The input resistance determines the sweep duration that produces the sharpest EPSP. A-D, Comparison of EPSP shape parameters for active models relative to the best fit parameters. Conductances were reduced to $1 / 3$ (black), 1/10 (blue), 1/30 (green), or 1/100 (gold) of their best fit values (magenta). The gray bar in $\boldsymbol{A}$ shows the threshold rate of rise (McGinley and Oertel, 2006). $\boldsymbol{E}$, The sweep duration that produces EPSPs with the fastest rise and largest amplitude (the optimal sweep duration) is plotted against the input resistance for passive models (black squares) and active models with best fit (open magenta triangles) or scaled membrane conductance (other colored symbols; key at bottom right). The black lines (and associated symbols) are for maximum amplitude; gray lines (and associated symbols) are for minimum half-width (key at top center). The sweep duration corresponding to maximum amplitude (dark lines) and minimum half-width (light lines) for active models with different membrane resistances or best fit passive models. For a 100 -fold range of membrane conductances, the input resistance spans an $\sim 25$-fold range, and the optimal sweep duration spans a range from 0.5 to $2.0 \mathrm{~ms}$. $\boldsymbol{F}$, To facilitate comparisons, the synaptic conductance for best fit passive or active models or membrane resistance-scaled models were adjusted for each cell so the EPSP for zero sweep duration had an amplitude of $15 \mathrm{mV}$.

each combination in each neuron, the remaining parameters were optimized to match the voltage traces using the same fit routine as for best fit simulations and achieving good fits in all cases (Table 1).

We were surprised to find that a wide range of parameter combinations gave EPSP shapes with sweep dependencies that were comparable with the best fit results. Varying membrane capacitance between 1.2 and $2.8 \mu \mathrm{F} / \mathrm{cm}^{2}$ and intracellular resistivity between 100 and $250 \Omega^{\star} \mathrm{cm}$ had little influence on the maximum rate of depolarization, maximum amplitude, and minimum half-width (Fig. 6A-C, left) or the associated optimal sweep durations (Fig. $6 A-C$, right). Differences across parameter combinations were comparable with the differences between cells in the best fit models (Fig. 6, error bars). Furthermore, EPSP shapes in these models were strikingly insensitive to variation in the soma/dendrite conductance ratio. Models with the smallest 

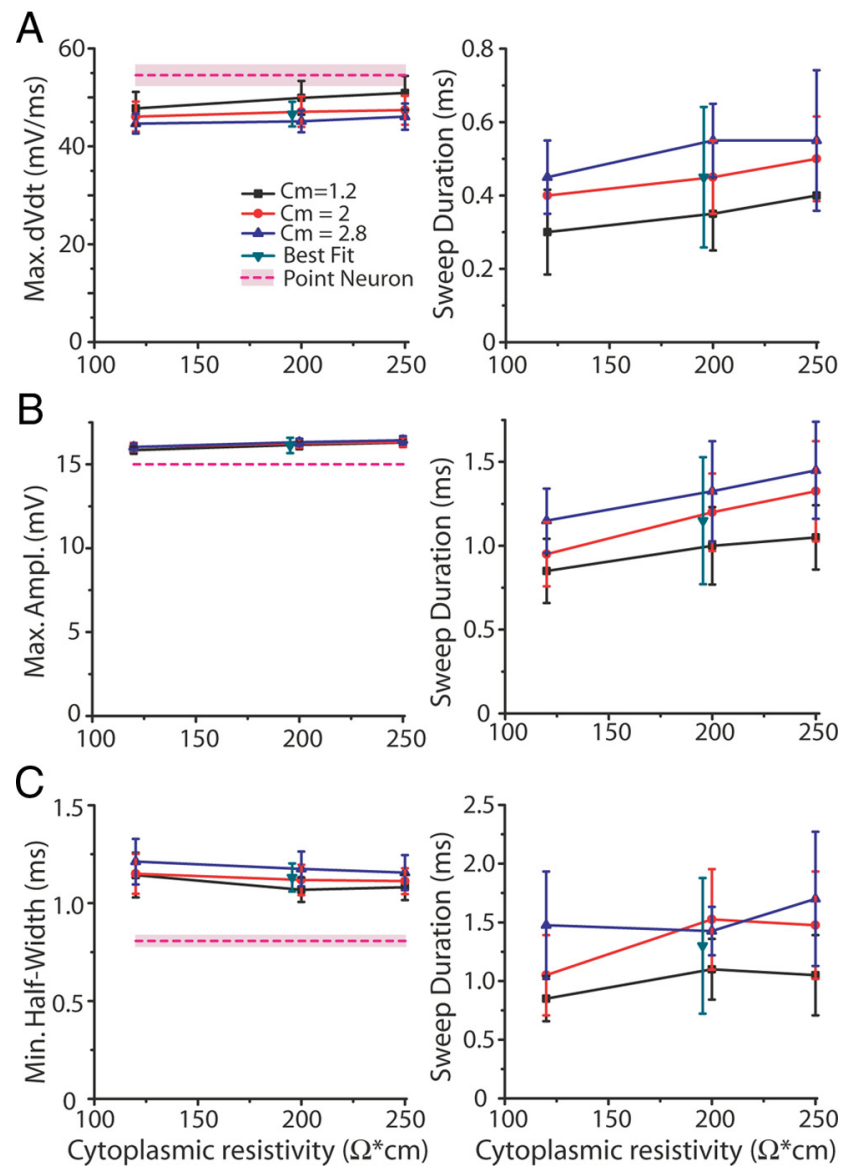

Figure 6. The dependence of the somatic EPSP shape on sweep duration is resilient to changes in the underlying model parameters. $A$, The magnitude of the maximum $d V / d t$ for the optimal sweep duration (left), as a function of intracellular resistivity. The pink band indicates \pm 1 SD. The sweep duration (right) corresponding to the EPSP with the maximum rising rate of depolarization ( $d V / d t)$ as a function of intracellular resistivity, for three values of the specific membrane capacitance. The green triangles are the average of the best fit result from each neuron. $\boldsymbol{B}, \boldsymbol{C}$, Maximum amplitude and minimum half-width, respectively. The plots have the same layout as in $A$. Integration windows follow the same patterns as the rate of rise (data not shown).

soma/dendrite conductance ratios, between 1 and 100, had almost indistinguishable EPSP shapes from best fit models, which had soma/dendrite conductance ratios between 400 and 35,000 (Fig. 7A). The maximum rate of rise depended weakly on the conductance ratio for large ratios (Fig. $7 B$, top). However, the sweep duration corresponding to the maximum rate of rise showed no dependence on the conduction ratio (Fig. $7 B$, bottom). Furthermore, the maximum amplitude and associated sweep duration did not depend on soma/dendrite conductance ratio (Fig. 7C). Therefore, the morphology, input resistance, and channel kinetics, but not the distribution of ion channels, govern the shape of EPSPs for soma-directed sweeps in octopus neurons, probably resulting from the consistently dominant contribution of synaptic membrane conductance regardless of passive membrane conductance gradients. This insensitivity to channel distribution is consistent with a previous observation in cortical neurons (Single and Borst, 1998). Our passive models were based on a hyperpolarizing regime that is outside the activation range of $g_{\mathrm{KL}}$ and in which the input conductance associated with $g_{\mathrm{KL}}$ at rest is compensated by the activation of $g_{\mathrm{h}}$ whose distribution may not be the same as of $g_{\mathrm{KL}}$.
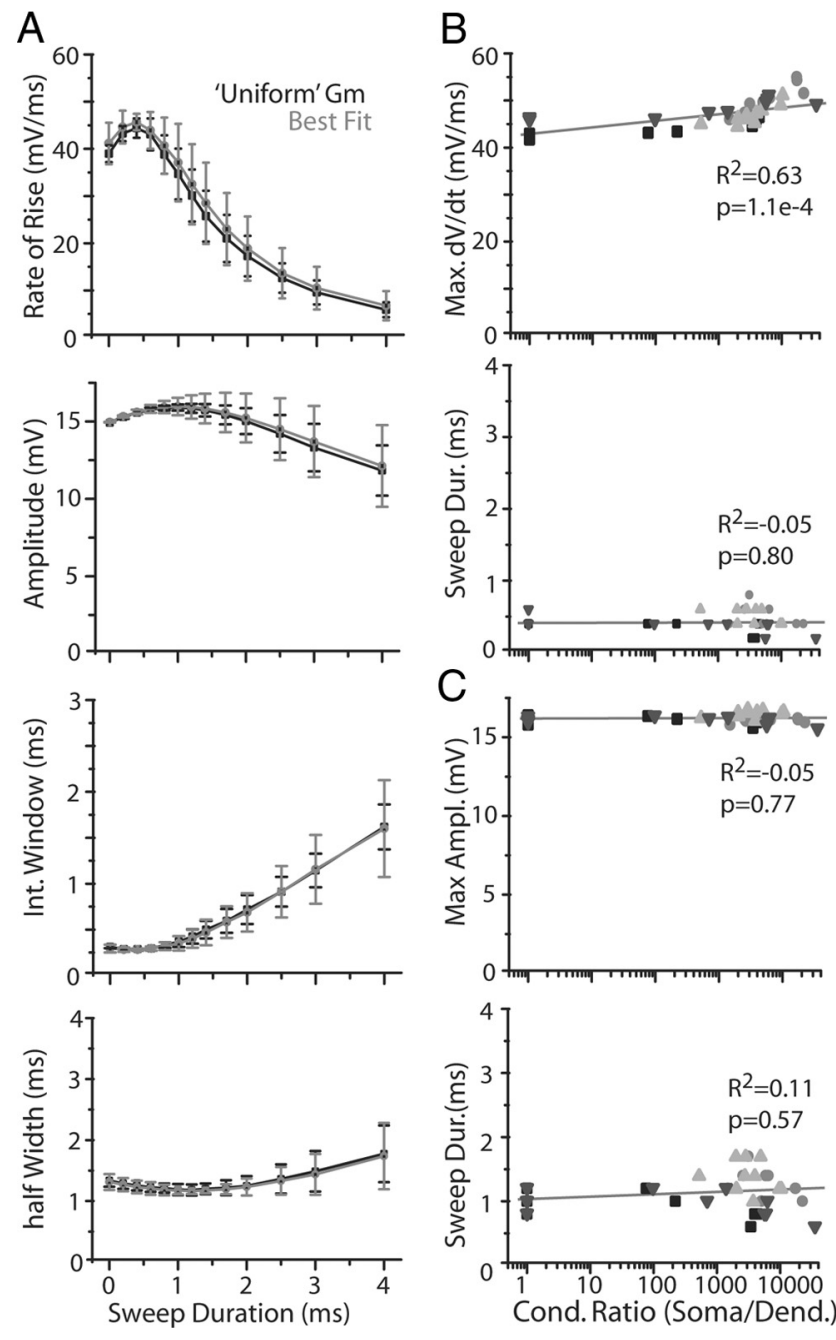

Figure 7. The somatodendritic distribution of ion channels minimally affects the shape of somatic EPSPs. $A$, Best fit models (gray) or models with the most uniform distribution of ion channels that still resulted in a good fit (black) did not have different EPSP shapes. For "Uniform Gm" models, two-cell models had equal somatic and dendritic conductance and two had a somatic-to-dendritic ratio of $\sim 100$. $\boldsymbol{B}$, The maximum rate of rise (top) and corresponding sweep duration (bottom) have only a slight dependence on the somatic-to-dendritic conductance ratio over a 10,000-fold range. $\boldsymbol{C}$, The maximum amplitude and corresponding sweep duration are also minimally sensitive to the channel distribution. Each shade/symbol corresponds to models of a single octopus cell. The gray lines are linear fits.

\section{Dendritic morphology and current spread}

Octopus cells were named on the basis of their striking morphology; dendrites emanate unidirectionally rostralward from the cell body (Fig. 8A, left). To understand the role of this morphology in sweep sensitivity, we analyzed the morphologies of the four digitally reconstructed octopus cells used in simulations (Fig. $8 \mathrm{~A}$, right). Octopus cells had substantial surface area in their dendrites. In particular, the surface area was distributed relatively uniformly over distance for a large proportion of their dendritic trees. This can be seen in a box representation of the morphology where the $y$-axis is the average total surface area and the $x$-axis is the average length for each branch order (Fig. $8 B$ ). There is substantial total surface area in the second through sixth branch orders, and even in the seventh and eighth orders (Fig. $8 \mathrm{~B}$ ). This distributed surface area results from progressively increasing the number while decreasing the diameter of the dendrites with distance from the soma (Fig. 8C).

Furthermore, octopus neurons have thick dendrites throughout their dendritic tree. The ratio of the diameter of the parent 
A

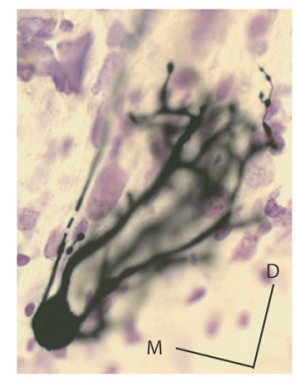

B
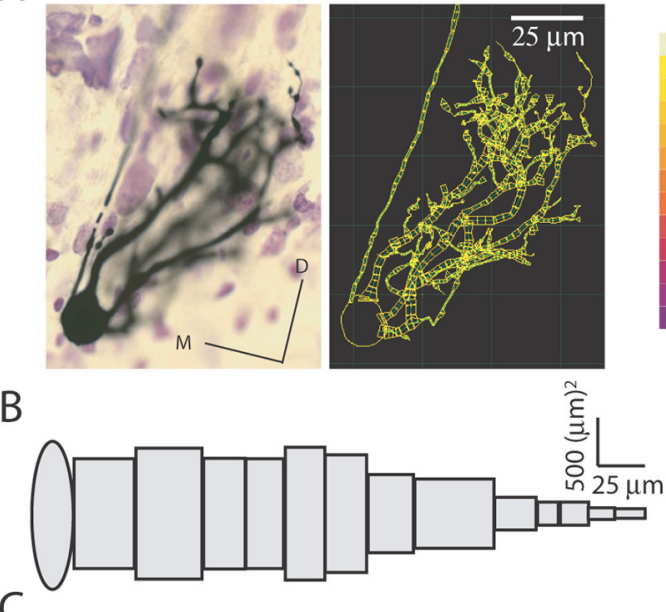

$E$

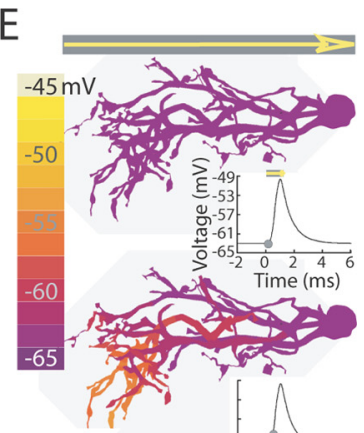

C
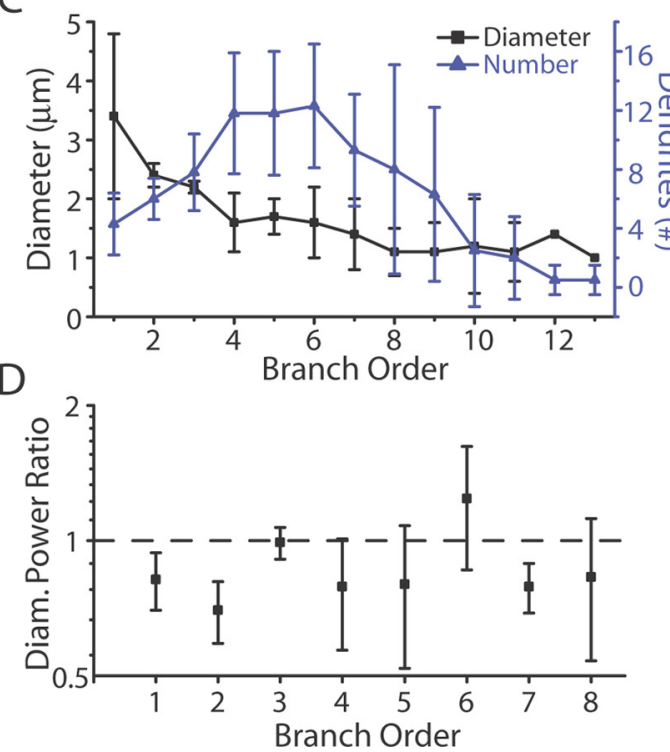

Figure 8. Octopus neurons distribute their surface area uniformly across distance. $A$, Photomicrograph of a labeled octopus cell (left) and 3D digital reconstruction of the same neuron (right). D, Dorsal; $M$, medial. $B, A$ box representation of the dendritic morphology of octopus neurons. Each box corresponds to a branch order. The $x$-axis is length, and the $y$-axis is total surface area. Each box is the average across cells of the average across branches within each order for each cell. The oval corresponds to the soma; the $x$-axis is the diameter, and the $y$-axis the surface area of a sphere with equivalent surface area to a reconstruction of the soma. C, The diameter (left, black) of dendrites decreases progressively, while the number of dendrites (right, blue) increases progressively with distance from the soma. $\boldsymbol{D}$, The dendritic diameter power ratio is $<1$ for most branch orders, including the first two. Dendrites were too small in diameter to accurately calculate the diameter ratio for branch orders $>8$. $\boldsymbol{E}$, Color maps of the spatial profile of the voltage for a sweep duration of $0.6 \mathrm{~ms}$ in an example simulated passive octopus cell. The time corresponding to each color map is indicated on the simulated EPSP traces (insets) as a green dot.

dendrite raised to the three-halves power divided by the sum of the diameters of the daughter dendrites raised to the three-halves power $\left(d_{i}^{3 / 2} /\left(d_{i+1,1}^{3 / 2}+d_{i+1,2}^{3 / 2}\right)\right.$, where $i$ indicates branch order, provides a measure of impedance matching between parent and daughter dendrites (Rall, 1964; Lanzinger, 1987; AgmonSnir and Segev, 1993). A ratio of 1 corresponds to matched impedance, and deviation from 1 results in impendance mismatch. In octopus cells, the branch diameter power ratio was $<1$ for most branch orders, resulting from large-diameter daughter dendrites (Fig. 8D). This will result in impedance mismatch at branches and thus contribute to dendritic propagation delays (Lanzinger, 1987). The resulting spread of depolarization down the dendrites of an octopus neuron during a $0.6 \mathrm{~ms}$ somatopetal sweep is illustrated in color maps of the voltage at several time points (Fig. $8 E$ ). During the rising phase of the EPSP, the tips of dendrites depolarize first, followed by the proximal dendrites, and then the soma (Fig. $8 E$, top five images), resulting in a rapidly rising EPSP (Fig. $8 \mathrm{E}$, insets). Subsequently, the entire neuron repolarizes (Fig. $8 E$, bottom two images).

\section{Discussion}

Octopus cells have unusually brief EPSPs and membrane time constants and fire with exceptional temporal precision (Oertel et al., 2000; Smith et al., 2005). Octopus cells are present in all mammals; in humans, octopus cells are large and prominent (Adams, 1997). Our results provide an explanation of why octopus cells have the morphology that gives them their name, and how this morphology helps them achieve their dramatic temporal precision. We show that octopus cells employ a combination of anatomical and biophysical features to detect the activation of synaptic inputs in soma-directed sweeps along their dendrites. Such soma-directed sweeps of inputs occur in responses to broadband transient sounds that are common in nature. It is also possible that soma-directed sweep sensitivity enables octopus cells to respond to frequencymodulated sweeps of sound (Rhode and Smith, 1986).

Our study shows that the predicted optimal sweep profile for exciting mouse octopus cells matches the cochlear traveling wave delay in mice. The differences in latencies to firing between auditory nerve fibers tuned to various frequencies that resulted from traveling wave delays was $1.6 \mathrm{~ms}$ (Fig. 1A). Individual octopus cells will not experience the full range of the traveling wave delay, because their dendrites extend over only part of the tonotopic axis. We can estimate the delay per octopus cell anatomically (Fig. 1). The tuning of auditory nerve fibers can be estimated, albeit roughly, from their location in the tonotopic axis. The tonotopic organization of the cochlear nuclei, like the cochlea, is a logarithmic function of the characteristic frequency (Liberman, 1982, 1991; Müller et al., 2005). In mice, the tonotopic axis in the posterior VCN is evident in the parasagittal plane (Wickesberg and Oertel, 1988). Auditory nerve fibers in mice are tuned to between $\sim 3$ and $80 \mathrm{kHz}$ (Taberner and Liberman, 2005). The tonotopic map, most clearly resolved in the anterior VCN where it is spread across the widest distance, was extrapolated to the octopus cell area by following the paths of labeled auditory nerve fibers. The octopus cell illustrated in Figure $1 B$ thus receives input from fibers with best frequencies between $\sim 4$ and $22 \mathrm{kHz}$ and would be expected to experience traveling wave delays spanning $\sim 0.6 \mathrm{~ms}$. Published images show that octopus cells vary in what proportion of the tonotopic axis their dendrites span, between $1 / 5$ and $2 / 3$, with octopus cells receiving the lowest frequency inputs spanning 
smaller ranges (Golding et al., 1995). Octopus cell dendrites are thus subjected to delays spanning between $\sim 0.3$ and $1 \mathrm{~ms}$ (average, $0.5 \mathrm{~ms}$ ). This range of delays matches the range of sweep durations that result in sharpest, most rapidly rising EPSPs in octopus neurons (Fig. 3).

Our results confirm that the simple, passive properties of a neuron can contribute to the sensitivity to soma-directed sweeps, as was shown in early theoretical work (Rall, 1964), and can perform a clear physiological function. We further show that the kinetics of $g_{\mathrm{KL}}$ and low input resistance tune the delay sensitivity to match the relevant physiological range. Ion channels that mediate $g_{\mathrm{KL}}$ reside in dendritic as well as in somatic membranes of octopus cells (Oertel et al., 2008). Cortical pyramidal cells exhibit sensitivity to soma-directed sweeps, but on a slower timescale and as a result of dendritic impedance gradients and nonlinear, NMDA receptor-mediated depolarization (Branco et al., 2010). Sensitivity to soma-directed sweeps may help shape receptive fields in visual cortical cells (Single and Borst, 1998; Anderson et al., 1999).

The mice used for in vitro measurements (P15-P17) are younger than those used for in vivo measurements (P28-P119). It is unlikely that maturation of the organization of the cochlear nucleus and of octopus cells affects our interpretations. First, the sample of octopus cells in this study did not differ detectably from other in vitro studies of somewhat older mice (P18-P26) (Golding et al., 1995, 1999; Bal and Oertel, 2000, 2001). Second, the hearing of $\mathrm{CBA} / \mathrm{J}$ mice, tested by distortion product otoacoustic emissions and auditory brainstem evoked responses, did not differ between 1- and 8-month-olds (Parham et al., 1999).

In mammals that hear at low frequencies, including humans, dogs, and cats, cochlear traveling wave delays are considerably longer than in mice, lasting $\sim 8 \mathrm{~ms}$ (Ruggero and Rich, 1987). These mammals have large, well developed octopus cells that are broadly tuned and fire with exceptional temporal precision (Osen, 1969; Adams, 1986, 1997; Rhode and Smith, 1986; Oertel et al., 2000). It is not known how individual octopus cells cope with the long traveling wave delays, whether their inputs span a larger range of delays, or whether there is a systematic difference in latency to firing across the population of octopus cells. The biophysical properties of these cells have not been examined in mature animals, but in puppies and kittens they are much like those in mice except that they have higher input resistances (Bal and Baydas, 2009; Bal et al., 2009). Our results show that increasing the input resistance of octopus cells increases the delay range to which they are most sensitive (Fig. $3 C$ ), providing a possible mechanism for compensation of longer traveling wave delays in animals with low-frequency hearing. The ubiquity and prominence of octopus cells in mammals raises the question, why they are needed? From what is currently known, we can only speculate. It is likely that the role of octopus cells concerns pattern recognition (Oertel and Wickesberg, 2002); the precise firing of octopus cells may serve to segment ongoing sounds and might help bind together the sound energies associated with a single source.

\section{References}

Adams JC (1986) Neuronal morphology in the human cochlear nucleus. Arch Otolaryngol Head Neck Surg 112:1253-1261.

Adams JC (1997) Projections from octopus cells of the posteroventral cochlear nucleus to the ventral nucleus of the lateral lemniscus in cat and human. Aud Neurosci 3:335-350.

Agmon-Snir H, Segev I (1993) Signal delay and input synchronization in passive dendritic structures. J Neurophysiol 70:2066-2085.

Anderson JC, Binzegger T, Kahana O, Martin KA, Segev I (1999) Dendritic asymmetry cannot account for directional responses of neurons in visual cortex. Nat Neurosci 2:820-824.

Bal R, Baydas G (2009) Electrophysiological properties of octopus neurons of the cat cochlear nucleus: an in vitro study. J Assoc Res Otolaryngol 10:281-293.

Bal R, Oertel D (2000) Hyperpolarization-activated, mixed-cation current $\left(I_{\mathrm{h}}\right)$ in octopus cells of the mammalian cochlear nucleus. J Neurophysiol 84:806-817.

Bal R, Oertel D (2001) Potassium currents in octopus cells of the mammalian cochlear nuclei. J Neurophysiol 86:2299-2311.

Bal R, Baydas G, Naziroglu M (2009) Electrophysiological properties of ventral cochlear nucleus neurons of the dog. Hear Res 256:93-103.

Branco T, Clark BA, Häusser M (2010) Dendritic discrimination of temporal input sequences in cortical neurons. Science 329:1671-1675.

Cao XJ, Oertel D (2005) Temperature affects voltage-sensitive conductances differentially in octopus cells of the mammalian cochlear nucleus. J Neurophysiol 94:821-832.

Cao XJ, Oertel D (2010) Auditory nerve fibers excite targets through synapses that vary in convergence, strength, and short-term plasticity. J Neurophysiol 104:2308-2320.

Cao XJ, Oertel D (2011) The magnitudes of hyperpolarization-activated $\left(I_{\mathrm{h}}\right)$ and low-voltage-activated potassium $\left(I_{\mathrm{KL}}\right)$ currents co-vary in neurons of the ventral cochlear nucleus. J Neurophysiol 106:630-640.

Clements JD, Redman SJ (1989) Cable properties of cat spinal motoneurones measured by combining voltage clamp, current clamp and intracellular staining. J Physiol 409:63-87.

Ferragamo MJ, Oertel D (2002) Octopus cells of the mammalian ventral cochlear nucleus sense the rate of depolarization. J Neurophysiol 87:2262-2270.

Gardner SM, Trussell LO, Oertel D (1999) Time course and permeation of synaptic AMPA receptors in cochlear nuclear neurons correlate with input. J Neurosci 19:8721-8729.

Gentet LJ, Stuart GJ, Clements JD (2000) Direct measurement of specific membrane capacitance in neurons. Biophys J 79:314-320.

Golding NL, Robertson D, Oertel D (1995) Recordings from slices indicate that octopus cells of the cochlear nucleus detect coincident firing of auditory nerve fibers with temporal precision. J Neurosci 15:3138-3153.

Golding NL, Ferragamo MJ, Oertel D (1999) Role of intrinsic conductances underlying responses to transients in octopus cells of the cochlear nucleus. J Neurosci 19:2897-2905.

Hines ML, Carnevale NT (1997) The NEURON simulation environment. Neural Comput 9:1179-1209.

Khurana S, Remme MW, Rinzel J, Golding NL (2011) Dynamic interaction of $I_{\mathrm{h}}$ and $I_{\mathrm{K}-\mathrm{LVA}}$ during trains of synaptic potentials in principal neurons of the medial superior olive. J Neurosci 31:8936-8947.

Kirkpatrick S, Gelatt CD Jr, Vecchi MP (1983) Optimization by simulated annealing. Science 220:671-680.

Lanzinger DJ (1987) Group delay caused by impedance mismatch. ARFTG Conference Digest-Spring 29:247-264.

Liberman MC (1982) The cochlear frequency map for the cat: labeling auditory-nerve fibers of known characteristic frequency. J Acoust Soc Am 72:1441-1449.

Liberman MC (1991) Central projections of auditory-nerve fibers of differing spontaneous rate. I. Anteroventral cochlear nucleus. J Comp Neurol 313:240-258.

Magee JC, Cook EP (2000) Somatic EPSP amplitude is independent of synapse location in hippocampal pyramidal neurons. Nat Neurosci 3:895-903.

Mainen ZF, Carnevale NT, Zador AM, Claiborne BJ, Brown TH (1996) Electrotonic architecture of hippocampal CA1 pyramidal neurons based on three-dimensional reconstructions. J Neurophysiol 76:1904-1923.

Maison SF, Emeson RB, Adams JC, Luebke AE, Liberman MC (2003) Loss of $\alpha$-CGRP reduces sound-evoked activity in the cochlear nerve. J Neurophysiol 90:2941-2949.

Mathews PJ, Jercog PE, Rinzel J, Scott LL, Golding NL (2010) Control of submillisecond synaptic timing in binaural coincidence detectors by $\mathrm{K}_{\mathrm{v}} 1$ channels. Nat Neurosci 13:601-609.

McGinley MJ, Oertel D (2006) Rate thresholds determine the precision of temporal integration in principal cells of the ventral cochlear nucleus. Hear Res 216-217:52-63.

McGinley MJ, Lazimy YM, Bal R, Oertel D (2005) Measurement and mod- 
eling of cable properties and optimal input delay profiles in octopus cells of the ventral cochlear nucleus. Assoc Res Otolaryngol Abs 444.

Müller M, von Hünerbein K, Hoidis S, Smolders JW (2005) A physiological place-frequency map of the cochlea in the CBA/J mouse. Hear Res 202:63-73.

Oertel D, Wickesberg RE (2002) Ascending pathways through the ventral nuclei of the lateral lemniscus and their possible role in pattern recognition in natural sounds. In: Integrative functions in the mammalian auditory pathway (Oertel D, Fay RR, Popper AN, eds), pp 207-237. New York: Springer.

Oertel D, Bal R, Gardner SM, Smith PH, Joris PX (2000) Detection of synchrony in the activity of auditory nerve fibers by octopus cells of the mammalian cochlear nucleus. Proc Natl Acad Sci U S A 97:11773-11779.

Oertel D, Shatadal S, Cao XJ (2008) In the ventral cochlear nucleus Kv1.1 and subunits of $\mathrm{HCN} 1$ are colocalized at surfaces of neurons that have low-voltage-activated and hyperpolarization-activated conductances. Neuroscience 154:77-86.

Osen KK (1969) Cytoarchitecture of the cochlear nuclei in the cat. J Comp Neurol 136:453-484.

Parham K, Sun XM, Kim DO (1999) Distortion product otoacoustic emissions in the CBA/J mouse model of presbycusis. Hear Res 134:29-38.

Rall W (1964) In: Neural theory and modeling (Reiss R, ed), pp 73-97. Stanford, CA: Stanford UP.

Rall W, Burke RE, Holmes WR, Jack JJ, Redman SJ, Segev I (1992) Matching dendritic neuron models to experimental data. Physiol Rev 72:S159S186.

Rapp M, Segev I, Yarom Y (1994) Physiology, morphology and detailed passive models of guinea-pig cerebellar Purkinje cells. J Physiol 474: $101-118$.
Rhode WS, Smith PH (1986) Encoding timing and intensity in the ventral cochlear nucleus of the cat. J Neurophysiol 56:261-286.

Ruggero MA, Rich NC (1987) Timing of spike initiation in cochlear afferents: dependence on site of innervation. J Neurophysiol 58:379-403.

Schmidt-Hieber C, Jonas P, Bischofberger J (2007) Subthreshold dendritic signal processing and coincidence detection in dentate gyrus granule cells. J Neurosci 27:8430-8441.

Single S, Borst A (1998) Dendritic integration and its role in computing image velocity. Science 281:1848-1850.

Smith PH, Massie A, Joris PX (2005) Acoustic stria: anatomy of physiologically characterized cells and their axonal projection patterns. J Comp Neurol 482:349-371.

Spruston N, Johnston D (1992) Perforated patch-clamp analysis of the passive membrane properties of three classes of hippocampal neurons. J Neurophysiol 67:508-529.

Stuart G, Spruston N (1998) Determinants of voltage attenuation in neocortical pyramidal neuron dendrites. J Neurosci 18:3501-3510.

Sugawara M, Murtie JC, Stankovic KM, Liberman MC, Corfas G (2007) Dynamic patterns of neurotrophin 3 expression in the postnatal mouse inner ear. J Comp Neurol 501:30-37.

Taberner AM, Liberman MC (2005) Response properties of single auditory nerve fibers in the mouse. J Neurophysiol 93:557-569.

Thurbon D, Lüscher HR, Hofstetter T, Redman SJ (1998) Passive electrical properties of ventral horn neurons in rat spinal cord slices. J Neurophysiol 79:2485-2502.

Wickesberg RE, Oertel D (1988) Tonotopic projection from the dorsal to the anteroventral cochlear nucleus of mice. J Comp Neurol 268:389399. 